with the word so interpreted it seemed easy to reconcile the narrative of St Luke with that of St Matthew xxvii 5. (2) The external evidence: the interpretation here suggested has the support of the Armenian Version and of the kindred Georgian Version. It is antecedently probable that the Armenian Version here depends on an Old Syriac text, and there is some evidence in Ephrem's Commentary on the Diatessaron that this was actually the case. This interpretation agrees with the tradition as to the death of Judas preserved by Papias, who possibly in the word $\pi \rho \eta \sigma \theta$ cis is consciously giving an equivalent of the Lucan phrase $\pi \rho \eta v i s^{\prime}$ yevómevos. The Old Latin and the Armenian renderings of $\pi \rho \eta v \epsilon \hat{\imath}$ in Wisdom iv 19 supply evidence either that $\pi \rho \eta v \eta^{\prime}$ bore the sense of 'swollen up' generally or that this was the significance attached to the word in Acts i I 8. Further, this interpretation of $\pi \rho \eta v$ ' in the Acts is certainly supported by a passage in the Acts of Thomas and probably by a fragment of Apollinarius on the death of Judas and by Athanasius's account of the death of Arius. The evidence so far is early evidence; and it comes to us, it will be noticed, from quite different quarters. The same interpretation is explicitly given by three late authorities-Georgius Cedrenus, Euthymius Zigabenus, and the Lexicon of Zonaras-each of whom is probably incorporating some earlier authority or some earlier tradition as to the meaning of $\pi \rho \eta \nu$ ís in Acts $\mathrm{i}$ I8.

F. H. ELY.

\title{
RICHARD CROKE'S SEARCH FOR PATRISTIC MSS IN CONNEXION WITH THE DIVORCE OF CATHERINE.
}

\section{De Richardi Croci (Yohannis Flandrensis) studiis Nazianzenicis.}

REM, quod sciam, uiris doctis non satis notam aggredior, de Gregorii Nazianzeni epistulis, quasnam in Henrici VIII, Britanniae regis, nota illa causa egerint partes, pauca quaedam moniturus.

Cuius quaestionis fontem epistulae praebent codicis Cotton. Vitell. B. XIII (s. XVI), quarum argumenta potissimum in opere Letters and Papers... of the Reign of Henry VIII ed. Brewer, tom. iv 3 (London 1876 ) extant edita, atque codicis Arundelliani I 5 I (s. XVI), in quem, dicti operis auctoribus ignotum, cum Nazianzeni inuestigarem libros, forte fortuna incidi. ${ }^{2}$

${ }^{1}$ Qui codex (in [T. Forshallii] libro Catal. of MSS in the British Museum : New series vol. I. [London] 1834 descriptus) plurium Croci epistularum continet apographa, maximam partem ab ipso Hieronymo Ghinuccio (uide infra) confecta, quae tunc praecipue, cum codex Cottonianus, secretioribus epistularum notis obscurus aut foedis lacunis laesus, deficit, maximo nobis sunt auxilio. 
Cum omnia Wolseii ipsius 'eiusque regis' frigerent consilia, neque quidquam Clementem VIII, Pontificem Maximum, inducere posset, ut regi Catharinam, quam, fratris morte uiduatam, uxorem duxit, matrimonio exigere permitteret, Cranmerus, ut uidetur, ${ }^{1}$ auctor exstitit, ut proprio Marte ac nomine uirorum doctorum, si qui regi fauerent, colligerentur sententiae et praecipue SS. Patrum excuterentur opera ad grauem illam controuersiam disceptandam, utrum Deuteronomii ${ }^{2}$ in hac causa adhibenda esset lex, an potius Leuitici,' qui fratris [mortui] uxorem in matrimonium ducere prohiberet. Nam ea quae iam prius a Wolseio collecta erant sententiarum 'magna uolumina' ad Pontificis Maximi tantum iura pertinebant interpretanda." Rex, quem propria 'conscientia' ne somnum quidem capere sinebat," grato animo hoc consilium est amplexus, utpote homo eruditus, ${ }^{\circ}$ quin. etiam ipse theologici cuiusdam opusculi ${ }^{7}$ auctor.

Itaque anno 1529 missus est hoc consilio in Italiam Richardus Crocus (Croke), uir Graece et Latine eximie doctus, qui cum Lutetiae Parisiorum antiquarum litterarum studiis in scholis Gulielmi Budaei et Hieronymi Aleandri ${ }^{\theta}$ operam dedisset, in Academia Lipsiensi (a. I514 et 1515-1517) et Cantabrigiensi (inde ab a. 1517) Graecae linguae exstitit sospitator, ${ }^{10}$ tunc autem Richmondiae Ducis erat informa-

1 J. A. Froude History of England from the Fall of Wolscy i, London 1875, pp. 267-269; H. A. L. Fisher The Political History of England, London, New York, Bombay 1906, v, pp. 287-288.

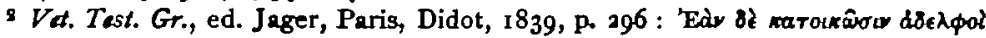

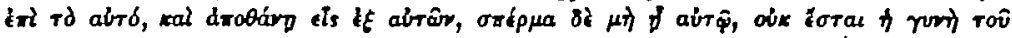

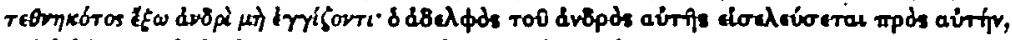

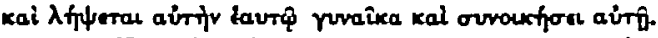

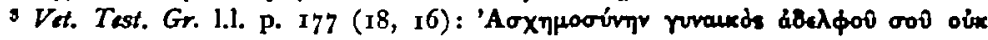

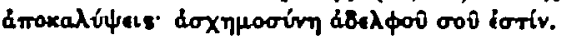

1 Brewer 1.1. Introd. pp. 258,267 .

- Brewer 1.1. Ep. 3140, 3643, 3644, 6111; cf. Introd. pp. 222-223.

- Cf. ex. gr. Brewer 1,1, Ep. 326I, 6I Ir.

T Libellus negivs (Assertio septem Sacramentorum) aduersus Martinum Lutherum hacresiarcham. Epistola rgia ad illustrissimos Saxomiae duces pia admonitoria. (Apud Londinium I52I.)

B Fisher 1.1.; Burnet The History of the Reformation of the Church of England, iii, Oxford I 865 , p. I 52 .

- Qui air doctus postea Bibliothecae Vaticanae praefectus (cf. infra), deinde episcopus (v. Brewer $E p .6159$ ) atque etiam cardinalis est factus; v. A. Mai Spriil. Rom. ii (1839) pp. 23I-233; G. Przychocki Watykahskie rekopisy listótw fwo. Graggona s Nasyansu (De Gregorii Nasianseni epistulanum codiabus Vaticanis), 'Eos' xvi (1910) P. IOI adn.

10 Specimen litteraturae Lipsicae, seculo xvi, in quo de Richardo Croco Britanno, Graecarum litterarum in Academiz Lipsica instauratore, exponitur ... praemissum a Iohanne Gottlob Boehmio, Lipsiae I 76r, pp. iv-xvi; Hager ap. Procentings of the Cambnidge Philological Saciety i-xxx, London 1893 , October term, 1882, November 2, pp. 33-35. Richardi Croci opera nota sunt haec : Ausomit editio, 1515 ; Theodon 
tor et magister. ${ }^{1}$ Iter Italicum ingrediens, praeceptis est instructus Iohan. Stokesleii, episcopi Londinensis, qui cum ei auctores proponeret examinandos, Nasianseni praecipue iussit quaerere' et perscrutari epistulas, nam caeca 'quadam ductus recordatione earum aliquam regis. causae admodum aptam et commodam esse credebat." Quo munere affectus Crocus, qui iam die 19 Decembris Bononiae erat et primum Patauinas et Veneticas bibliothecas inspicere, dein autem Romam proficisci in animo habebat, ${ }^{4}$ omne fere tempus Venetiis in libris euoluendis consumpsit. Quae non tam iucunda ei euenit prouincia, nam non solum omnium bibliothecarum ac praecipue Marcianae ${ }^{5}$ difficillimos aditus, sed etiam complurium hominum, qui Pontificis Maximi partibus fauerent, adeo infestos inuenit animos, ut, ne regis cognosceretur minister, Iohannis Flandrensis nomen sibi imponere debuerit. ${ }^{\circ}$ Accedebat, quod etiam cum Iohannis Casalis, oratoris regii,' decertandum ei erat invidia ac simultate. Hic enim, ut Croco inueniendae epistulae laudem prae; riperet, nihil sibi praetereundum duxit, quo eum ad rem perficiendam impediret; ueluti, si Croco fides, adulterinum ei obtulit Marcianae bibliothecae catalogum, in quo Gregorianos codices de industria omisisset. Attamen patricii cuiusdam Veneti, Georgii Francisci (quem summis extollit laudibus atque saepius regiae commendat liberalitati), eiusque nepotis uiri docti Domitii Treuisani opera, Crocus non solum uerum Marcianae bibliothecae impetrauit indicem, e quo duos Gregorianarum epistularum codices in hac bibliotheca exstare comperit, ${ }^{10}$ sed mox etiam ipsius bibliothecae adeundae copiam est adeptus. Nam licet etiam die 6 Ianuarii conqueratur 'de bibliotheca publica Veneta nihil possum impetrare, nec scio quorum medio ducem et senatum

[Gasan] libri IV editio, Lipsize I516; M. R. Croci Londoniensis Tabulas, Graccas litteras comperdio discere cupientibus same quam wtiles..., Lipsiae 1516 ; Orationes Rich. Croci duac, altora a cura, qua mtilitatom laudemque Graecae linguae tractat, altera a tempore, qua horlatus est Cantabrigimses, me desertores essent ciusdem I 520.

1 Brewer Ep. 4562 .

1.Nondum enim omnes erant editae, cum editio priaceps, anno 1528 confecta (Basilï Magni at Gngorii Nasianseni Theologomm Epistulal Graces, rumquam antea edita. Opus plame sandwm a theologicum. Haganose per loh. Sec[erium]), 57 tantum Gregorianas contineret epistulas. Tunc tamen, ne haec quidem editio uiris doctis, de quibus agitur, nota fuisse uidetur, nam Aldinam tantum commemorant Graecarum epistularum editionem. (Epistolae diversac philosophonwm, etc. ... Venetiis apud Aldum ... M I D. V. Brewer $E p .6{ }_{1} 8$ et Aldinae editionis praefationem. Cf. praeterea Brewer $E_{p}$.6rga.)

- Brewer Ep. 6105, 6149,6165, 6192, 6205, 6229, 6251.

- Brewer Ep. 6161.

- Brewer Ep. 6140. Brewer Ep. 6168. T Burnet 1.1. pp. I53-153.

- Casalis peruersitatem passim conqueritur Crocus, sed uideas praecipue apud Brewerum Epp. 6229, 6235, 6236.

- Brewer Ep. 6r 746236.

10 Brewer $E_{p}$. 6165, 6r68, 6173, 6r92, 6r97, 6229, 6235, 6280. 
Venetum ea de re sollicitarem', ${ }^{1}$ iam die 28 eiusdem mensis haec scribit :

'Hodie fuimus apud Sanctum Marcum et in ipsa bibliotheca capsas nonnullas et in eis graconum plenam quandam invenimus, in qua multa erant graeca egregia opera tum oratorum, tum vero Philonis et Chrisostomi et Dionysii. Invenimus etiam librum epistolarum graecarum maximum volumen, sed auctoris nomen extritum, cum indagaremus (?) eum, reddidit nos dubios utrum Basilii an Nazianzeni ; fuisse Basilii, nam erant ad monachos aliquot epistolae.'s

Quid, si non ita multo post, etiam ut domum libros examinandos acciperet, impetravit?

'Heri [die 3 Febr.], opera patris Francisci domi permittebantur Canones conciliorum cum commentis in Graeco ... postremo Nasianzeni epistolarum volumina, sed pulchrum illud cum Basilii epistolis, de quo antea scripsi [Brewer $E p .6$ r 73 ], heri non erat in capsa, ubi id reliqueram. Policiti sunt procuratores, habiturum me ubi istos restituero.'

Sed Crocus ne ceteras quidem, quae hoc tempore Venetiis extabant, praetermisit bibliothecas: etenim priusquam ipsam Marcianam inspexit, iam eiusdem Francisci Georgii auxilio SS. Iohannis et Pauli peruestigauit bibliothecam," de qua die 6 Ian. haec scripsit: 'Inueni etiam in Bibliotheca Iohannis et Pauli Nazianzenum sed non epistolas, inueni etiam Basilii epistolas.' “ Attamen postea, ut ex alia quadam epistula, die 2 Martii scripta, elucet, 'antiquissimum Gregorii Nazianzeni epistularum ' hic inuenit ' codicem'. Cuius bibliothecae libri anno ${ }_{789}$ in Marcianam sunt delati, ${ }^{7}$ sed in Castellanii catalogo, qui libros m. s. post annum 1740 illatos recenset, ${ }^{8}$ nullus nunc SS. Iohannis et Pauli commemoratur codex, qui Gregorii Nazianzeni contineat epistulas. At etiam a. 1740 Dominicus M. Berardelli huius bibliothecae codicem quendam $n$. LXI signatum, chartaceum, s. circa xul, his uerbis

1 Brewer Ep. 6140= cod. Arund. I51 fol. 144"-145". CE. Brewer Ep. 6168.

- Haec ex optime seruato codicis Arundelliani (fol. I64-I65) apographo attuli, cum in codice Cottoniano et ipsa Croci epistula (Brewer $E p .6156$ ) et eius apographum, notis secretis conscriptum (Brewer Ep. 6173), permultas exhiberent lacunas. Cf. Brewer Ep. 6165 .

Quae ex ipso codice Cottoniano (fol. $3^{2}$ ) deprompsi, nam apud Brewerum (Ep. 6197) argumentum solum exstat anglice conscriptum. CE. Brewer Ep. 6165, 6192,6238 .

4 Brewer Ep. 6192, 6239. De hac quoque bibliotheca libros domi legendos accepit. V. Brewer Ep. 6149 .

- Brewer Ep. 6140, cf. supra adn. I. Brewer Ep. 6250.

7 T. Valentinelli Bibliotheca Manuscripta ad S. Marci Veneliarum. Cod. MS Latini I Venetiis 1868 , c. xiii pp. $96,98 \mathrm{sq}$.

- C. Castellani Catalogus Codicum Graccornm, qui in bibliothecam D. Marci Venetianum inde ab anno MDCCXL ad hare usque tompora inlati sunt. Venctiis 1895. 


\section{$\therefore \quad . . .$. NOTES AND STUDIES ... . . : 289}

describit : 'Nazianzeni (S. Gregorii) Epistolae 14.7, Galeni de diebus

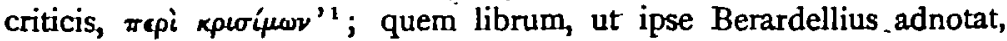
etiam Montefalconius (a. 1702) in Diario Italico (p. 47) commemorauit : 'Codex XII saeculi. Epistolae Gregorii Nazianzeni et quaedam Basilii.' (Cf. Bibl. Bibl. i 478.) Ad eundem fortasse librum, cum inter omnes constet illius aetatis in codicibus describendis indiligentia, ea pertinent, quae apud Tomasinium (a. 1650$)^{2}$ de quodam eiusdem bibliothecae

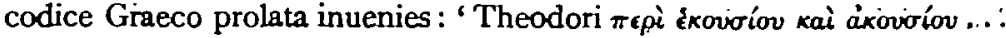
Georgii Gemistii de uirtute ... Basilii et aliorum epistulae, Galenus de diebus criticis.' Ex iis autem, quae hic de Theodori [Gazaei] et Georgii Gemistii [Plethonis] operibus sunt dicta, non sine aliqua probabilitate conici potest hunc esse illum librum $\mathrm{m}$. $\mathbf{s}$. quem, ipsius Croci aetate (s. xvi) exaratum, commemorat codex Vaticanus gr. 2240 (Column. 79),

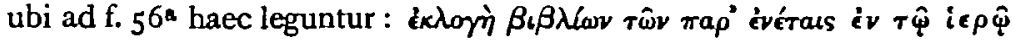

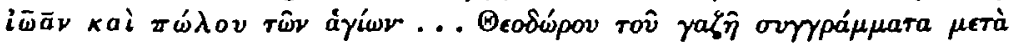

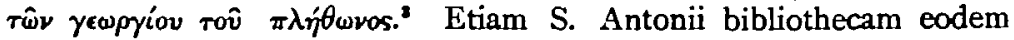
Francisco Gregorio intercedente Crocus inspexit "; quae bibliotheca a Grimaniorum familia condita erat (Tomasini l. 1. p. I) ad eamque sine dubio referenda sunt, quae in Croci epistula, die 4 Febr. ad Ghinuccium episcopum Wigornensem ${ }^{3}$ scripta, leguntur: 'Iam audio non a protonotario solo, sed etiam a pluribus aliis fide dignis, habere Cardinalem Grimanum et epistolas Nasianzeni omnes et Basilii et concilia omnia graeca cum epistolis et decretis et canonibus hic Venetiis.' Codicum tamen, qui in hac bibliotheca asseruabantur-obscura prorsus sunt fata, nam a. 1687 Bibliotheca tristi incendio est consumpta, neque ullum librum seruatum esse Castellanius contendit (l. l. p. 44 adn.). At tamen Leo Dores, ignotis quibusdam documentis innisus, sane probabilem protulit opinionem plures huius bibliothecae codices aut ipso incendii tempore aut iam prius surreptos esse eoque modo in alias bibliothecas atque in ipsam Marcianam peruenisse. ${ }^{7}$ Quibus liceat mibi adicere aliud quoddam, a Morellio adhibitum testimonium, e quo, ut opinor, eluceat partem saltem eorum librorum in Marcianam bibliothecam re uera transisse: 'Poco dopo [intellege past annum $15^{89}$ ]

1 Dominicus Maria Berardelli, ' Codicum omnium Graecorum . . . qui manuscripti in bibliotheca S. Iohannis et Pauli ... essservantur, catalogus' ap. Nrova Raccolta - d' opuscoli sciontifici efilologici t. $\times x(1740)$ fol. K. 5 .

1. Ph. Tomasini Bibliothecas Venetae mankscriptae ... Utini 1650, 'Bibliotheca SS. Ioannis et Pauli' pp. 22-23.

- Heiberg Philal. 55 (18g6) p. 746 sq.

- Brewer Ep.6174.

- Qui erat regius orator Romae, atque omnium Henrici VIII ministrorum quodammodo magister ac princeps, ut ex his omnibus epistulis elacet.

a Brewer Ep. 6197, cr. supra p. 288 adn. 3.

7 Reuke Critique 30 (1896) p. 91-92.

VOL. XIII 
secondo un Decreto del Senato I636. 24 Nov. lasciob Libri alla Republica anche il Patriarca d'Aquileja Giovanni Grimani, da cui ebbe il Publico Museo gran parte d' Anticaglie : ma più chiara notizia non ritrovo, che dia conferma a questo fatto.' ${ }^{1}$ Qua re efficitur, ut etiam huius bibliothecae libros, etsi a Castellanio (1. l.) non designatos, in Marciana liceat quaerere. De illis autem codicibus, quos, ut suspicor, Crocus, Gregorianas quaerens epistulas, legisse potuerit, tres praecipue apud Tomasinium (1. 1) uideas :-

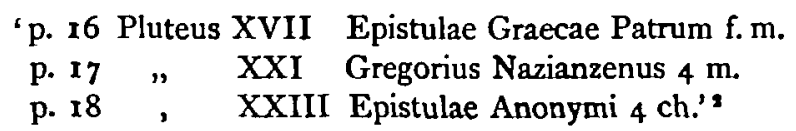

Patauinam denique Crocus inspexit S. Iustinae bibliothecam, in qua tamen Nazianzeni orationes solas inuenit, non epistulas. ${ }^{3}$

Iam uideamus quae Crocus his omnibus bibliothecis et codicibus excussis eruerit et quatenus munus sibi delatum praestiterit : Gregorianam quidem epistulam, quam ita comparatam habere uoluit Stokesleius ut regis causam adiuuaret, etsi tantos subiit labores, non inuenit; et profecto inuenire non poterat hanc solam ob causam, quod eiusmodi epistula Gregoriana omnino nulla est. Quid, si in eius epistulis, ad aliquod matrimonium pertinentibus ( $E p p$. 144, 145, 193, 230, 231, 232), 目 nonnulla extant eis, quae Stokesleius moliebatur, prorsus contraria,

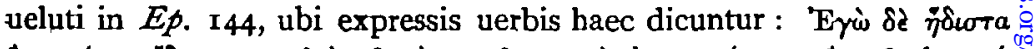

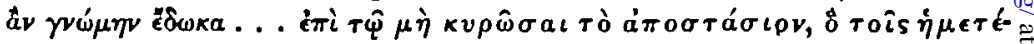

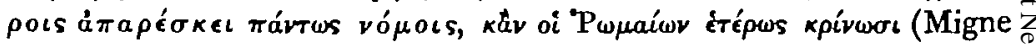
$P$. Gr. 37, col. 245, 248)? Itaque non mirabimur, quod apud regios ministros orta sit suspicio, huic quaestioni fortasse errorem quendam Stokesleii " subesse, praesertim cum I. Casalis eiusmodi epistulam se non inter Gregorianas, sed. inter Basilianas legisse praedicaret. ${ }^{5}$ Nunc igitur Crocus omne studium ad Basilianas epistulas examinandas conuertit et breui epistulam illam, in qua totius causae cardo uertebatur, Basilii epistulam 199 (Migne, 1. 1. 32, col. $724 \mathrm{~A}$ ), re uera inuenit. Haec enim legas, quae ex Croci epistula, die r8 Febr. (?) ad regem missa excerpsi :-

- Basilius ille Neocaesariensis, Invictissime et potentissime princeps, qui ob exi[miam religi]onem et pietatem et magnus et sanctus non immerito dictus est, in libro ad Amphilochium episcopum ita graece

1. I. Morelli Della Pubblica Libreria di S. Marco in Venesia Dissortatione storica, Venezia 1774, pp. xxxviii-xl, iii.

2 V. praeterea de hac bibliotheca : Blume It. Ital. p. 225.

- Brewer Ep. 6149; 6168 (p. 2756), cf. I. Ph. Tomasini Bibl. Patavinas MSS, Utini 1639 , pp. 14, xi-xii.

- Brewer Ep. 6170, cf. Ep. 6r59.

4. Brewer Ep. 6194, 6174. 


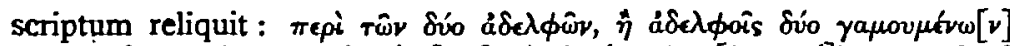

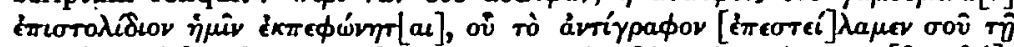

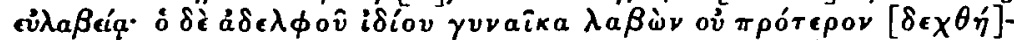

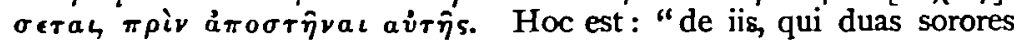
duxere, aut [duobus] fratribus nupsere epistolula a nobis edita est, cuius adversarias misi ad pietatem tuam. Ceterum qui proprii fratris uxorem duxerit, non prius admittatur in fidelium communionem, quam ab ea fuerit separatus." Ex quibus verbis non temere divino (sic) hanc esse illan, quam nos tantopere quaerimus et lapsum memoria Stoxleium Nasianseno ascripsisse, quod era $[t]$ Basilii. Eam igitur ad maiestatem tuam mittimus ...'

\section{Dein Franciscum Gregorium laudare pergit :-}

' Hic solus . . hic Antonianam bibliothecam mihi aperuit. Hic Antonianae et Marcianae indices veros, sine quibus ficticium illum orat[oris] tui numquam deprehendissemus, mihi tradidit describendos. Hic domino Dominico $\operatorname{Tr}[$ evisano] nepoti suo, homini graece et latine exacte docto persuasit, ut sua fide et nomine primum quidem ex Iohannis et Pauli deinde ex Sancti Marci Bibliotheca libros desumeret, quos domi meae perlegendos permitteret. Et hi sunt illi [venerandae?] antiquitatis graeci libri, in quibus epistolam tam diu nobis quaesitam invent[mus].' 1

Quibus tamen Crocus minime sibi acquiescendum esse putauit et, ut quaestioni omnem adimeret scrupulum, iterum et saepius Gregorianas uersauit epistulas; tunc etiam unam et alteram, si quae omnino ad matrimonium referri poterat, ad Ghinuccium mittebat, ut ex eius litteris ad Stokesleium datis elucet :-

' Superioribus his diebus ad te misi epistulam Basilii, quam tu as[cripsisti] Nazianzeno. Evolvi autem Nazianzeni epistulas omnes, nec aliquam inveni, quae utcunque ad nostrum negotium vel suspicione posset trahi, praeter duas, quas quamvis huc videantur nihil pertinere, ut ne indiligentem in evolvendis libris putes, descriptas ad episcopum misi."

Atque huc pertinet ex.c. Greg. Naz. Ep. 232, quam primo miserrime mutilatam, dein integram inuenit, ut ipse dicit :-

'Legimus epistulas Nazianzeni omnes et nihil in his, quos habemus, libris invenimus, sed in altero antiquissimo S. Iohannis et Pauli invenimus epistulam integram, cuius mancam partem misimus, scriptamque eam ad Dioclem, non quod putabamus ad Amphilochium." Nec ulla in omnibus istis Nazianzeni praeter haric et alteram, quae ulla suspicione

1 Cod. Cotton. fol. $47(48)^{\circ}$. Apud Brewerum (Ep. 6229) argumentum solum inuenitur.

3 Cod. Cotton. fol. $5^{8}$, Brewer $E_{p} .625 \mathrm{I}$.

- De Gregorii Nazinnzeni epistula 232 agitur, cuius fragmentum in litteris die 22. Febrr. ad Ghinuccium datis laudauit, V. Brewer Ep. 6239 
ad nuptias trahi possent, et eas quamquam nihil ad rem facientes, ut ne tamen videamur fuisse indiligentes, mi[ttimus].'1

Iam igitur ad certum erat redactum Basilianam solam, quae huc faceret, esse epistulam.

Alia nunc oritur quaestio nondum profligata, utrum Crocus Romae rewera fuerit, id quod principio in animo habebat (u. supra), necne? Atque primum est, quod moneamus, omnino non uideri uerisimile, ut Crocus, Henrici VIII minister, Pontificis Maximi, qui omnia regis nouerat consilia, bibliothecam adire, nedum excutere potuerit, etiamsi Poenitentiarii illud officium adeptus fuisset. Et profecto in nulla epistula Croci, Ghinuccii aut aliorum, qui huic causae operam dabant, testimonium aliquod inuenimus eiusmodi, ut eo innisi Crocum aliquando Romae fuisse ac bibliothecam Vaticanam inspexisse credamus; quin contra his epistulis perlectis, pro explorato necesse est habeamus Crocum ipsum breui sibi persuasisse nullam in Vaticana bibliotheca spem poni debere. Quae Croci sententia luculentissime apparet ex Ghinuccii quadam epistula ad regem missa, quam in breuiorem formam redactam-cum etiam ad Vaticanae bibliothecae historiam non sine momento uideatur esse-ex codice Arundelliano ${ }^{\circ}$ affero :-

'Scribit Crocus ex Venetiis valde longas litteras, datas ro. octavo Ianuarii ${ }^{\circ}$; quod ad rem attinet in substantia est invenisse se Venetiis Bibliothecarium Papae [Hieronymum Aleandrum] ${ }^{7}$ ab eoque intellexisse

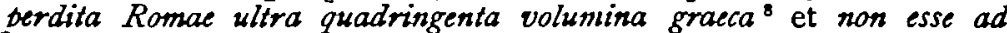
praesens in Bibliotheca librumi illum Nazianzeni, quem quaerimus, sed iactare apud se totum esse Nazianzenum licet non ibi sed in Apulia item dictum sibi fuisse a quodam Valeriano Burgomense [Bergamo] ' uiro docto, esse apud episcopum Theatinum [Chieti] ${ }^{10}$ ibi existentem

1 Cod. Cotton. fol, $57\left(5^{8}\right)$. Quae a Brewero $(E p .6250)$ perperam sunt intellecta.

'Cf. Encyclopadia Britannica ${ }^{10}$ s.u. 'Croke Richard' et Allgemeine Dentsche Biographic iv, Leipzig 1876, pp. 602-604 (Horawitz).

- Brewer Ep. 6108, p. 2757.

4 Cf. Brewer Ep. 6ro5.

- In fol. $15^{2}-160^{\circ}$ ea exstant, quae ad Crocum pertinent, Ghinuccii manu ut uidetur scripta, prius quam ea notis secretioribus condidit; quae recensio lectu difficilis et multis lacunis foeda apud Brewerum (1.1. Ep. 6r68) exstat.

- Brewer Ep.6149.

T V. supra p. 286, cf. Brewer Ep. 6149, 6150.

8 Cf. Brewer Ep.6149, 615o. Quae ad famosam illam urbis Romae occupationem (anni 1527) quae sacco di Roma dicta est, sine dubio sunt referenda. Attamen de ' 400 libris omissis' neque E. J. Vogel Gasch. d. Vaticana ssit d. Zrit Sixtus IV. bis \&. J. 1600 (Seraperm vii, 1846), pp. 289 sq., neque E. Mantz La Bibliothique du Vatican an $X V T^{*}$ siecte (Pdite Bibl. d' art at d' archeol. Paris 1886 ), ullam faciunt mentionem, Fr. Blume autem (Itrr. Ital. pp. 27I-272) Vaticanam Bibliothecam in hac calamitate criguo aut omnino nullo damno affectam esse asseuerat.

- Non 'Burgi ...' ut Brewer in suo codice se legisse putat; cf. Ep. 6193, 6149.

10 I. Petrum Carafnm dicit, qui postea Pontifex Maximus Paulus IV factus est, hoc autem tempore, post urbem captam, Venetiis erat. Cf. Brewer Ep. 6r50, 6173. 
non solum .Nazianzenum, sed etiam Maximum Planudem èt Crisostomum et rapsodias multorum commentariorum in utrumque Testamentum et fere omnes libros quos dominus Stoksleyus ex mandato maiestatis vestrae dixit sibi esse legendos ... petiit etiam a me consilium, utrum deberet ulterius ibi manere ... ego autem ad eum sc[rip]si; quod mihi videbatur eum debere ibi manere, donec speraret posse cau[sae] maiestatis vestrae prodesse, nam Romae non videfur esse tantas spes [ut spera]bamus...'

Atque iam die 2 Febr. ipse Crocus haec scripsit :-

'... Iam certum est hic libros omnes illos esse, quos mihi Romae legendos praescripsit Stoxleius, et Nazianzeni epistulas illas, quas nos maxime cupimus ; et Romae non esse et hic esse a fide dignis . . . accepimus.' 1

Cum denique ex posterioribus Croci epistulis aperte eluceat eum de Vaticana Bibliotheca, cuius custos Aleander regi esset inimicissimus, prorsus desperauisse, ${ }^{2}$ atque idem Crocus in alia quadam epistula a rege petat, ut se Venetiis in Germaniam mittat," procliuis est coniectura Crocum epistula illa Venetiis inuenta iam de Roma nibil curauisse et breui alio quodam munere esse affectum. Adde, quod non ita multo post ipsa regis causa iam peracta erat.

Ultima autem epistula, quam in hac causa ex Italia misit Crocus (Brewer $E p .6745$ ), Venetiis est scripta et postea, anno demum r 532, eum deprehendimus, Oxoniensem professorem, regis libellum de suo diuortio conscriptum " summis efferentem laudibus."

At ex bibliotheca Vaticana est depromptum opusculum quoddam Graecum, quod in cod. Arundelliano i $5 \mathrm{I}$ asseruatur (fol. $187^{\mathrm{a}}, \mathrm{r} 88^{\mathrm{b}}$ ) atque his lemmatibus est instructum : ' Ex puplica bibliotheca ponteficis ex libro, qui inscribitur Collecta B. Gregorii Nazianzeni in fine tertii banchi supra.' Et paulo infra: 'Epistula inventa in libro Gregorii Nazianzeni in bibliotheca ponteficis in tertio banco.' Huc sine dubio pertinet Ghinuccii epistula, cuius argumentum in eodem codice Arundell. (fol. $183^{\mathrm{a}}, 184^{\mathrm{a}}$ ) asseruatur : 'Excerpta ex litteris Domini Wigorniensis die vii Februarii Bononiae datis.' Cum epistula ipsa apud Brewerum

Eius libri autem in Vaticanam bibliothecam postea sunt delati. Cf. V. Gardthausen Sammlungen u. Cataloge griech. Hss., Leipzig 1903 [Byz. Archiv. 3], p. 44.

1 Cod. Cotton. fol. 296, cf. Brewer Ep. 6rg2.

2 Brewer Ep. 6280 (21 Mart.) p. 2823; 6354 (23 Apr.). Quod hic (p. 2853) apud Brewerum 'Alexander' pro 'Aleander' legitur, merus error est.

3 Cod. Cotton. fol. $49^{\circ}$ ( $\left.50^{\circ}\right)$ haec a Croco scripta habet : '. . . Si regi placet et videtur mihi non posse melius facere, quam si amicos quidem parandos huic patri. [Francisco Gregorio], libros graecos evolvendo[s] Dominico Trevisano eius nepoti [i]n regem propter litteras (l) optime fideliterque affecto (?), graece praeterea cxacte docto, committeret et nos in Germaniam ad parandos illos amicos mitteret.'

1 A glasse of the truthe [London 1531 ].

- Brewer 1.1. vol. v $E$ p. 1338, cf. Ep. 1320 et I 180. 
ex cod. Cottoniano edita (ep. 6205) admodum sit mutilata, haec uelim legas e codice Arundelliano descripta :-

' Mittit [Ghinuccium intellege] copiam quorundam scriptorum Graecorum ex quodam volumine Gregorii Nazianzeni ex bibliotheca pontificis et ex illo banco, quod dixerat Dominus Stokleus, in quo libro est illa. epistula, quae quacrebalur. Et unus Graecus dixit, quod dicta scripta non continebant quae quaerebamus, tamen quod faciunt aliquid contra adversarios nostros, sed tutius istic, quam hic veritas epistulae interpretabitur [1], cuius etiam exemplum misi ad dominum Crocum.'

Quae tamen noua et sane mira "epistula" e duobus constat capitibus :

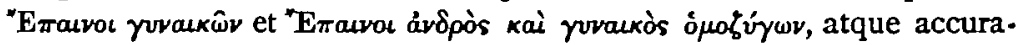
tius examinata non nisi cento quidam e Gregorii Nasianzeni orationibus conflatus apparet. Nam haec uelim uideas, quae ex. c. ex ipso principio

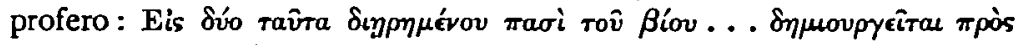

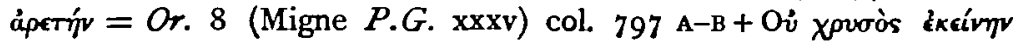

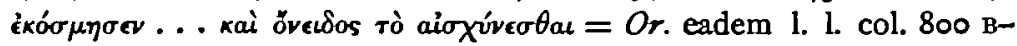

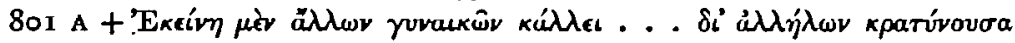

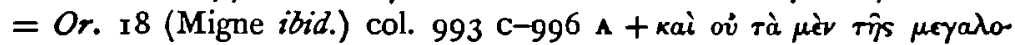

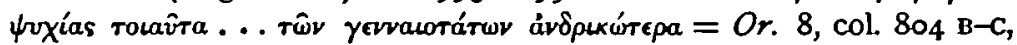
et q. $\mathrm{s}$.

Quid si etiam codicem Vaticanum, e quo haec essent descripta, inuenisse contigit? Hic est codex Vat. 485 s. xiii, qui titulo 'Collecta B. Gregorii Nazianzeni et Maximi' denotatus, Anthologiam continet Gregorianam, in qua (in capitibus $\overline{\boldsymbol{\epsilon}}-\mathrm{v} \overline{5}, \mathrm{pl} .88^{\mathrm{b}}-\mathrm{go}^{\mathrm{b}}$ ) eadem prorsus atque in codice Arundelliano extant.9 Itaque uides nescioquem-caue ne Crocum putes-lucri fortasse faciendi studio ductum, haec ex libro Vaticano excerpsisse atque iis, qui Gregorianas quaererent epistulas, pro uera Nazianzeni epistula obtrusisse, aut saltem obtrudere studuisse.

1 Cf. [Forshall, J.] Catal. of MSS in the Brit. Mus. New series [London] vol. i : The Arundel MSS 1834, p. 40: 'De hoc opusculo mithil traditur in editione operum Gregoni Nasiamsmi Parisiis impressa a. 1788 studio Monachorum S. Mauri.' Minime mirum id quidem.

- De hoc codice aliisque duobus a me examinatis, qui eandem contineant Anthologiam, u. Przychocki Eos xvi (rgro) pp. log-I $1_{4}$ (cf. supra, p. 286 adn. 9). Cum autem reliqui duo codices (Pii II 7 , Vat. gr. 484 $_{4}$ ) posteriore demum saeculi xvi dimidio essent exarati, codicis Vat. 485 solius hic rationem esse habendam suspicabar. Quam coniecturam Monsignor I. Mercati, a me rogatus, ut est eximia comitate, per litteras ad me missas ad certum redegit. Qui uir doctus praeterea hunc codicem a Sixti IV temporibus $\left(14 j^{1-1484}\right)$ re vera 'in tertio banco' fuisse asseruatum benigne me docuit. Aperte autem conspirant, quae de Platina, Sirti IV Biblio. thecario, in libro Acta litteraria ex manuscriptis eruta atque collocta cura Burcardi Gotthelffi Struveri fasc. IV, Ienae 1706, p. IO, sunt dicta: ' Dividit Platina libros secundum banca sive repositoria, ita, ut primum bancum contineat Biblia ... tertium alia adhuc Augustini, Ambrogii, B. Gregorï. . . . 
Quae omnia autem luculenter demonstrant, quanta etiam hac detate Gregorii Nazianzeni fuerit auctoritas, ut in hoc tam graui discrimine ad eius praecipue opera uiri docti prouocauerint.

Gustavus Przychocki.

Scribebam in uico Gromnik.

\section{ORIGEN'S COMMENTS ON THE APOCALYPSE.}

IT was only in July last that a Greek scholar of the University of Athens, M. Diobouniotis, sent to Dr Hamack the copy of a text of a considerable part of the Apocalypse accompanied by the comments of some early patristic writer whom he was not able to identify. The copy was taken from a tenth-century manuscript in the monastery of Meteoron, which had already pielded a work of Hippolytus, 'On the blessings of Jacob.' Harnack at once recognized this new commentary as the work of Origen, with the exception of the last two comments which were extracts from Irenaeus known to us hitherto for the most part in Latin only. With characteristic speed Harnack produced an editio princeps which was in our hands before the middle of December. ${ }^{1}$ Students of Origen, and students of the text of the Apocalypse, must of necessity read this little tract for themselves; so that I shall not take up space by any further description of its contents. I only ask leave to make a few emendations of a text, which, notwithstanding the excellent work that Harnack has done upon it, still challenges the corrector.

I. Scholion xi (p. 26) 1. 6. This sentence is quite right as it stands. It means: Perhaps his perplexity arises from thinking of ordinary death; whereas what has been said above as to 'the second death' may fairly be applied, if need be, to an angel.

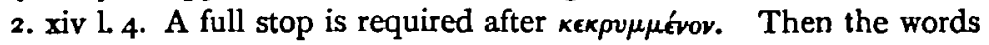

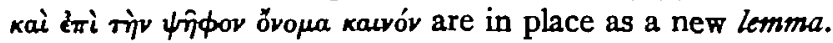

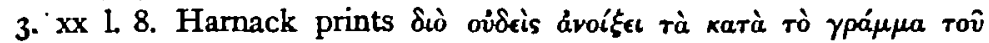

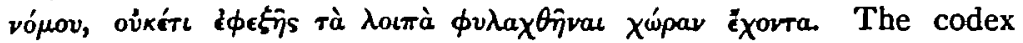

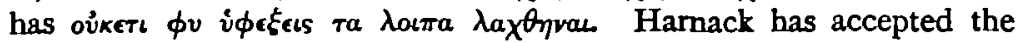
ingenious correction offered by Diobouniotis; but he adds 'at locus nondum sanus est'. It seems quite clear that in the archetype a line

1 Der Scholien-Kommentar des Origenes zur Apokalypse Johannis, nebst einem Stack aus Irenaeus, Lib. $\nabla$, Graece, entdeckt und herausgegeben von Constantin Diobouniotis und Adolf Harnack (Texte und Untersuchungen, 191 I). 